\title{
Quantitative Analysis of the Ultrasonic Vocalization Responses Elicited in Adjuvant-Induced Arthritic Rats for Screening Analgesic Drugs
}

\author{
Hiroyuki NAITO and Maki INOUE \\ Department of Physiology II, School of Veterinary Medicine, Azabu University, \\ Fuchinobe, Sagamihara 229-8501, Japan
}

\begin{abstract}
Adjuvant-induced arthritic (AIA) rats have been developed as a chronic pain model to evaluate the effects of analgesic drugs. The purpose of the present study was to examine whether there is dose-dependent inhibition of the emission of ultrasonic vocalization (USV) responses by analgesic drugs in AIA rats. It was demonstrated that morphine (1.25-5.0 mg/ $\mathrm{kg}$, s.c.) and ketoprofen (2.5-10.0 mg/kg, s.c.) dose-dependently inhibit USV responses. These results suggest that the USV responses elicited in AIA rats are useful for the quantitative evaluation of analgesic drugs.
\end{abstract}

Key words: adjuvant-induced arthritic rats, ketoprofen, morphine, naltrexone, ultrasonic vocalization

Freund's complete adjuvant-induced arthritic (AIA) rats have been developed to evaluate the effects of analgesic drugs as an experimental model of chronic pain in many studies [2, 3, 6, 7]. Calvino et al. [1] and Jourdan et al. [4] reported that the ultrasonic vocalizations (USVs) emitted by AIA rats during social interactions were inhibited by analgesic drugs. Therefore, it appears that the USVs emitted during social interactions by AIA rats may be considered as an expression of affective pain.

In a previous study [5], we demonstrated that AIA rats emit USVs in the 22-28 kHz range with 300-1,200 ms duration when they were confronted with a 'stimulus rat' during social interactions. In addition, the USVs emitted by AIA rats were found to be significantly inhibited by three analgesic drugs (piroxicam, rofecoxib, and ketoprofen), whereas diazepam, an anxiolytic benzodiazepine receptor agonist, did not cause such inhibition. The dosages used are known to have antinociceptive properties in conventional screening methods. Thus, a direct relationship between chronic pain and USV response evoked in AIA rats is strongly suggested.

The purpose of the present study was to examine whether quantitative analysis of the USV response in AIA rats could be achieved using two analgesic drugs to establish a new screening method using the same paradigm.

Male Lewis (LEW/CrlCrlj) arthritic rats weighing $210-260 \mathrm{~g}$ were individually housed in cages in an airconditioned room $\left(22 \pm 2^{\circ} \mathrm{C}\right)$ with the lights on from 07:00 to 21:00 and were given free access to water and food directly available on the floor. The AIA rats were tested 21 days after Freund's complete adjuvant injection into their right hindpaws. All procedures in this study

(Received 9 October 2007 / Accepted 11 March 2008)

Address corresponding: H. Naito, Department of Physiology II, School of Veterinary Medicine, Azabu University, Fuchinobe, Sagamihara 229-8501, Japan 


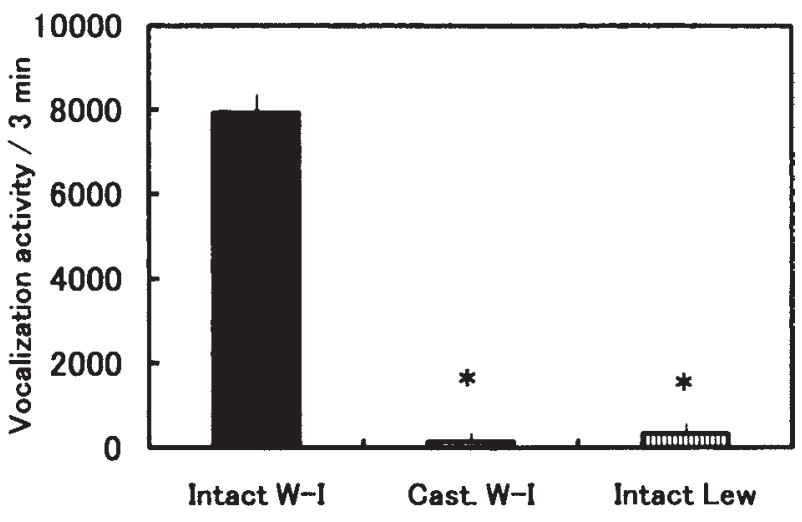

Fig. 1. Ultrasonic vocalizations in adjuvant-induced arthritic Lewis rats $(\mathrm{n}=8)$ when confronted with intact WistarImamichi (W-I) male rats, castrated W-I male rats, and intact Lewis male rats, 'stimulus rats' during social interaction. All data were analysed by means of the MannWhitney $\mathrm{U}$ test. Asterisks denote significant difference $(P<0.01)$ from intact W-I male rats.

were approved by the Animal Research Committee of Azabu University.

In the preliminary experiment, three intact WistarImamichi (W-I) male rats weighing 430-460 g, three W-I male rats castrated at 3 weeks of age (410-430 g), and three intact Lewis male rats (430-460 g) were compared with regard to their aptitude as 'stimulus rats' for the social interaction tests. As indicated in Fig. 1, intact W-I male rats were shown to elicit stable and high USV responses from AIA subjects. Intact Lewis male rats and castrated W-I rats were less effective. A significant difference in vocalization activity was observed between intact W-I rats and castrated W-I and intact Lewis rats $(P<0.01)$. However, no significant difference was observed between castrated W-I and intact Lewis rats. In our previous study, we noticed that the vocalization activity of AIA rats is largely influenced by the locomotor activity of the 'stimulus rat'. Vigorous locomotor activities of intact W-I rats may elicit more ultrasonic vocalizations when compared with castrated W-I and intact Lewis rats. We, therefore, used intact W-I male rats for the 'stimulus rats' in the present study.

During the preliminary experiments, we observed that AIA subjects spontaneously displayed signs of submission including USVs when placed in a neutral test cage containing one of the 'stimulus rats'. The procedure for the elicitation of USV responses in AIA subjects by the social interaction test was the same as that previously described [5]. 'Stimulus rat'-elicited USV responses in AIA subjects were measured for 3 min around 11:00 in the morning. Two hours later, morphine or ketoprofen was injected. Measurement of USVs was then repeated 30 and 60 min after the injection. Morphine hydrochloride (Shionogi \& Co., Ltd., Osaka, Japan) was injected subcutaneously at a dose of $1.25-5.0 \mathrm{mg} / \mathrm{kg}$ body weight. Ketoprofen (Capisten, Kissei Pharmaceutical Co., Ltd., Nagoya, Japan), which is a prostaglandin-endoperoxide synthase (Ptgs-1 (PTGS1) and Ptgs-2 (PTGS2)) inhibitor, was injected subcutaneously at a dose of 2.5-10.0 $\mathrm{mg} / \mathrm{kg}$ body weight. Moreover, the effect of the pretreatment of naltrexone hydrochloride $(1.0 \mathrm{mg} / \mathrm{kg}$, i.p., Sigma Chemical Co., MO, USA), an opioid receptor antagonist, $10 \mathrm{~min}$ before morphine injection $(5.0 \mathrm{mg} / \mathrm{kg}$, s.c.) on the USVs was examined. USVs were measured $30 \mathrm{~min}$ after morphine injection, and their appropriate vehicles were injected subcutaneously or intraperitoneally to control groups in a volume of $2 \mathrm{ml} / \mathrm{kg}$ body weight. The effects of the drugs on the USV responses were expressed quantitatively as the percentage inhibition of vocalization activity after injection as compared to that before injection (\% inhibition) in each subject. The effects of the drugs on the USV responses were analyzed using Wilcoxon's matched-pairs signed-ranks test and the Mann-Whitney U test. Significance was defined as the 0.05 level.

The effects of the two analgesic drugs on the USV responses elicited from AIA subjects are shown in Fig. 2. Morphine (Fig. 2A) exhibited a dose-dependent inhibition of USV responses at 30 and $60 \mathrm{~min}$ after the injection [\% inhibition (mean $\pm \mathrm{SEM}$ ): $1.25 \mathrm{mg} / \mathrm{kg}: 30$ $\mathrm{min}, 17.3 \pm 3.9 \%, 60 \mathrm{~min}, 35.2 \pm 4.8 \% ; 2.5 \mathrm{mg} / \mathrm{kg}: 30$ $\min , 46.4 \pm 4.9 \%, 60 \mathrm{~min}, 56.8 \pm 4.9 \% ; 5.0 \mathrm{mg} / \mathrm{kg}: 30$ $\min , 98.6 \pm 0.8 \%, 60 \mathrm{~min}, 100.0 \pm 0 \%$; vehicle: $30 \mathrm{~min}$, $1.8 \pm 1.8 \%, 60 \mathrm{~min}, 4.4 \pm 1.9 \%$ ], and significant dosedependent effects were found among the dosages $(P<0.05)$. Ketoprofen (Fig. 2B) also exhibited a dosedependent inhibition of USV responses at 30 and $60 \mathrm{~min}$ after injection [\% inhibition (mean \pm SEM): $2.5 \mathrm{mg} / \mathrm{kg}$ : $30 \mathrm{~min}, 17.5 \pm 2.7 \%, 60 \mathrm{~min}, 18.4 \pm 1.8 \% ; 5.0 \mathrm{mg} / \mathrm{kg}$ : $30 \mathrm{~min}, 47.8 \pm 2.8 \%, 60 \mathrm{~min}, 38.4 \pm 2.2 \% ; 10.0 \mathrm{mg} / \mathrm{kg}$ : $30 \mathrm{~min}, 87.6 \pm 1.1 \%, 60 \mathrm{~min}, 78.0 \pm 3.3 \%$; vehicle: 30 min, $-0.65 \pm 3.5 \%, 60 \mathrm{~min}, 0.9 \pm 3.3 \%$ ], and significant 

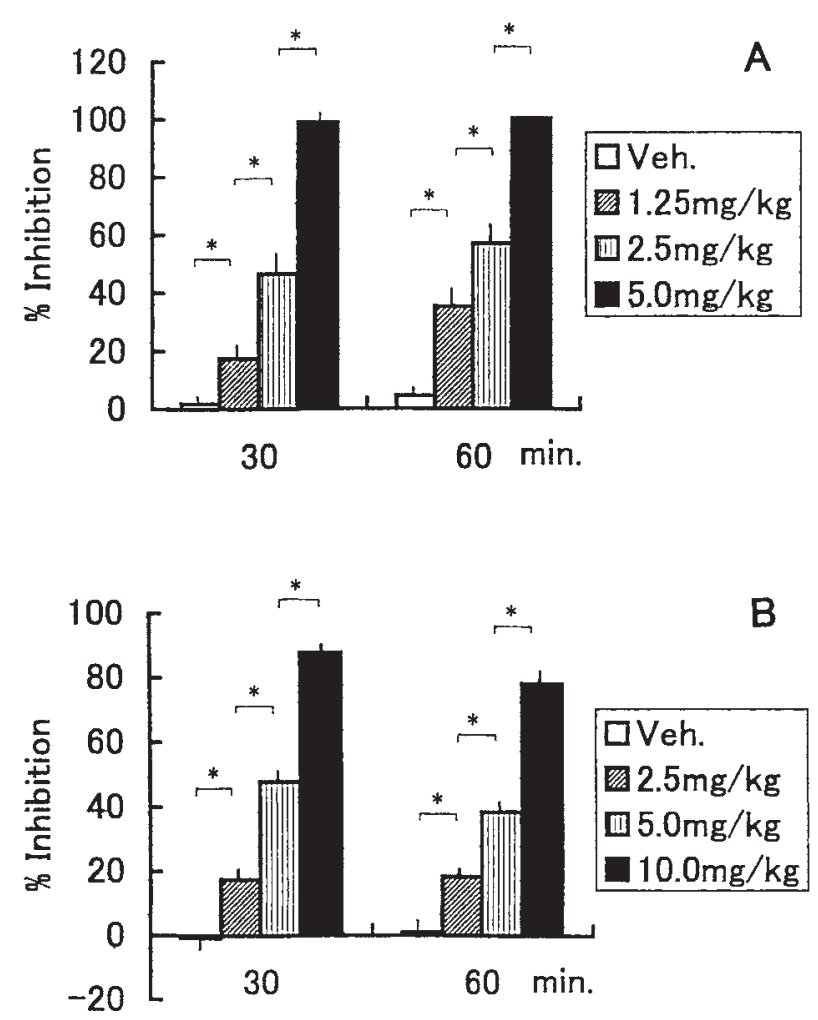

Fig. 2. Effects of morphine (A) and ketoprofen (B) on USV responses in adjuvant-induced arthritic rats during social interaction. The effects of the drugs on USV responses were expressed quantitatively (mean $\pm \mathrm{SEM}, \mathrm{n}=8$ ) as percentage inhibition of vocalization activity after injection as compared to that before injection in each subject. All data were analysed by means of Wilcoxon's matched-pairs signed-ranks test. Asterisks denote significant differences $(P<0.05)$ among the dosages.

dose-dependent effects were found among the dosages $(P<0.05)$. The effect of the administration of naltrexone hydrochloride before morphine injection on the USVs is shown in Fig. 3. Naltrexone significantly antagonized the inhibition of the USVs induced by morphine $(P<0.05)$ [naltrexone + morphine: $14.8 \pm 4.2 \%$; vehicle + morphine: $96.8 \pm 0.7 \%$ ]. Naltrexone had no effect on the USVs [naltrexone + vehicle: $3.8 \pm 3.2 \%$ ]. Calvino et al. [1] have also reported that morphine ( $3 \mathrm{mg} / \mathrm{kg}$, i.p.) decreased the USVs elicited in AIA rats during social interactions, though they did not verify the dose-response relationship.

Morphine is known to have an anxiolytic action because Sánchez [8] has reported an inhibition of footshock-induced USVs. However, in our previous study

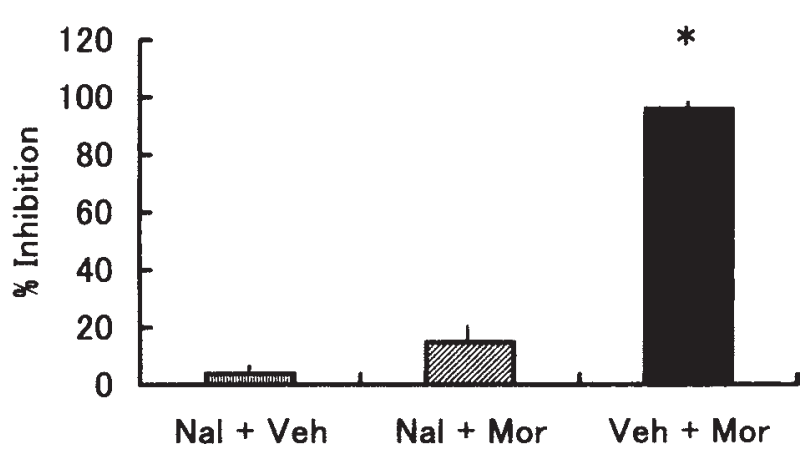

Fig. 3. Effects of morphine (Mor., $5.0 \mathrm{mg} / \mathrm{kg}$, s.c.) on the USV responses in adjuvant-induced arthritic rats with or without pretreatment with naltrexone ( $\mathrm{Nal} ., 1.0 \mathrm{mg} / \mathrm{kg}$, i.p.). Pretreatment with naltrexone was done $10 \mathrm{~min}$ before morphine injection. The effects of the drugs on USV responses were measured $30 \mathrm{~min}$ after morphine injection and expressed quantitatively (mean \pm SEM, $n=6$ ) as percentage inhibition of vocalization activity after injection as compared to that before injection in each subject. All data were analysed by means of Wilcoxon's matched-pairs signed-ranks test. The asterisk denotes significant difference $(P<0.05)$ from Nal. pretreatment groups.

[5], diazepam, an anxiolytic benzodiazepine receptor agonist, did not inhibit USVs elicited in AIA subjects during social interaction tests. Calvino et al. [1] also reported that aspirin, a peripheral anti-inflammatory and analgesic compound with no anxiolytic properties, suppressed USVs elicited in AIA rats during social interaction. Therefore, the USV responses investigated in the present study are likely to be associated with chronic pain. Ketoprofen was used because it is clinically effective in chronic pain and because we confirmed the inhibition of USV emissions after injection of this drug in our previous study [5]. The present results confirm that the USVs recorded during social interaction are dosedependently inhibited by the administration of analgesic drugs to AIA rats.

At the present time, besides AIA rats, there is no experimental model for chronic pain that is appropriate for eliciting USV responses, and ethical measures must be adequately considered when we use this experimental model.

In conclusion, quantitative analysis of the USV response as an indicator of chronic pain in AIA rats might be more advantageous for screening analgesic drugs than those of conventional screening methods. 


\section{Acknowledgment(s)}

We wish to thank Y. Fukuda for his skillful technical assistance.

\section{References}

1. Calvino, B., Besson, J.M., Boehrer, A., and Depaulis, A. 1996. Neuroreport 7: 581-584.

2. De Castro Costa, M., De Sutter, P., Gybels, J., and Van Hees,
J. 1981. Pain 10: 173-185.

3. Hirose, K. and Jyoyama, H. 1971. Jpn. J. Pharmacol. 21: 717-720.

4. Jourdan, D., Ardid, D., and Eschalier, A. 2002. Pain 95: 165-173.

5. Naito, H., Okumura, T., Inoue, M., and Suzuki, Y. 2006. Exp. Anim. 55: 125-129.

6. Okuyama, S. and Aihara, H. 1984. Jpn. J. Pharmacol. 34: 67-77.

7. Pircio, A.W., Fedele, C.T., and Bierwagen, M.E. 1975. Eur. J. Pharmacol. 31: 207-215.

8. Sánchez, C. 2003. Eur. J. Pharmacol. 463: 133-143. 